\title{
A rare case of Nocardia and non-tubercular mycobacteria co- infection of lung, bone and joints in a young female
}

\author{
Dipankar Pal*, Arindam Naskar, Soumyadip Chatterji, Manab Kumar Ghosh, Shekhar Pal, Dushyant Lahre and Amartya Kumar Misra \\ Department of Tropical Medicine, School of Tropical Medicine, Kolkata, India
}

\begin{abstract}
Both Nocardia and non-tubercular mycobacteria are opportunistic human pathogens. Co-infection is rare and not found in the literature. We detected a young female suffering from such a co-infection in both lungs, joints and bones. She subsequently improved on simultaneous treatment for both of these organisms.
\end{abstract}

\section{Introduction}

Nocardia is a soil borne saprophytic, aerobic actinomycete causing either localised infection of lung or wide spread disseminated infection particularly to the brain, soft tissue and skeletal structures. Nocardia asteroides is the main human pathogen (85\%); N.brasiliensis, N.otitidiscaviarum are the other main pathogenic nocardiae. Nocardiosis chiefly affects immunocompromised hosts such as patients with lymphoreticular malignancies; solid tumours, transplant recipients, HIV infection, alcoholism, diabetes mellitus, trauma, prior granulomatous diseases like tuberculosis, connective tissue diseases like sarcoidosis, systemic lupus erythematosus [1].

Non-tubercular mycobacteria (NTM) are widely distributed in the environment with high isolation rates worldwide [2]. Organisms can be found in soil and water, including both natural and treated water sources (M.kansasii, M. xenopi, and M. simiae are recovered almost exclusively from municipal water sources and rarely, if ever, fromother environmental sources). Human disease is suspected to be acquired from environmental exposures, although the specific source of infection usually cannot be identified. NTM may cause both asymptomatic infection and symptomatic disease in humans including lung, soft-tissues etc. Joints, bones, tendons are usually affected by local inoculation of the pathogen by direct trauma or surgery [3]. Occasionally, axial bones or extremities may be affected by NTM without apparent trauma.

\section{Case report}

A 27 years old non-diabetic female presented with low-grade evening rise of temperature, productive cough, anorexia and significant weight-loss for last one and a half years. It was soon followed by swelling of both her ankles, right elbow and both wrist joints along with dorsum of feet and hands. All the sites were tender and there was blood mixed sero-sanguinous discharge from the swelling of hands and feet. Her complaints underwent a waxing and waning course with chronic ill-health during this time-period.

She developed left sided tubercular pleural effusion three years back and received two months of ATD (rifampicin, isoniazid, pyrazinamide, ethambutol). But with partial recovery she stopped her treatment prematurely. Pleural effusion recurred after one year for which she again received ATD for eight months with complete cure this time.

On-examination, she was pale, cachectic, with swelling of dorsum of both hands (Figure 1a), right wrist (Figure 1b), right elbow (Figure 1c), dorsum of right foot (Figure 2), right ankle. Joint temperature was normal with restricted movement and sero-sanguinous discharge was noted from all the involved joints. Spleen was palpable $3 \mathrm{~cm}$ below left costal margin. Coarse crackles were present in the right infraclavicular region.

Chest $\mathrm{x}$-ray (Figure 3) revealed in non-homogenous opacities involving both upper zones and right mid-zone. X-ray right hand (Figure 4a) and right foot (Figure $4 \mathrm{~b}$ ) revealed osteolytic lesions. Sputum and wound-discharge from multiple sites including both hands and feet for acid fast bacilli (AFB) were positive. Gram stained smear of sputum showed gram positive beaded, branched organisms suggestive of nocardia (Figure 5). Culture grew non-tubercular mycobacteria (NTMs- Mycobacterium abscessus) and Nocardia asteroides from the discharge of hands and feet. HIV serology was negative and she was not on any immunosuppressive medication.

In addition to surgical wound debridement treatment was started with oral co-trimoxazole, co-amoxiclav for Nocardia and clarithromycin, amikacin and imipenem for NTM as per Infectious Disease Society of America (IDSA) guidelines [4]. After one month, fever subsided with reduction of cough, expectoration and joint

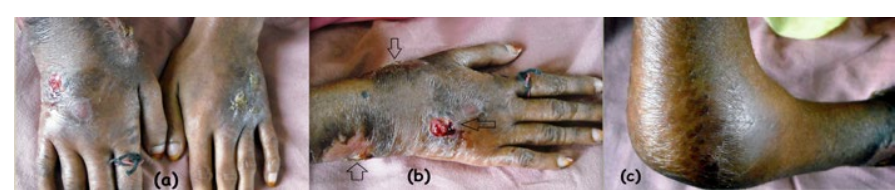

Figure 1. Lesions of (a) both hands, (b) right wrist and (c) right elbow at presentation.

Correspondence to: Dipankar Pal, RMO cum Clinical Tutor, Department of Tropical Medicine, School of Tropical Medicine, Kolkata, India, Tel: +919432113713, E-mail: dipankarpal.2009@gmail.com

Key words: nocardia, non-tubercular mycobacteria, co-infection, pulmonary disease, osteomyelitis

Received: August 01, 2015; Accepted: September 15, 2015; Published: September 18, 2015 


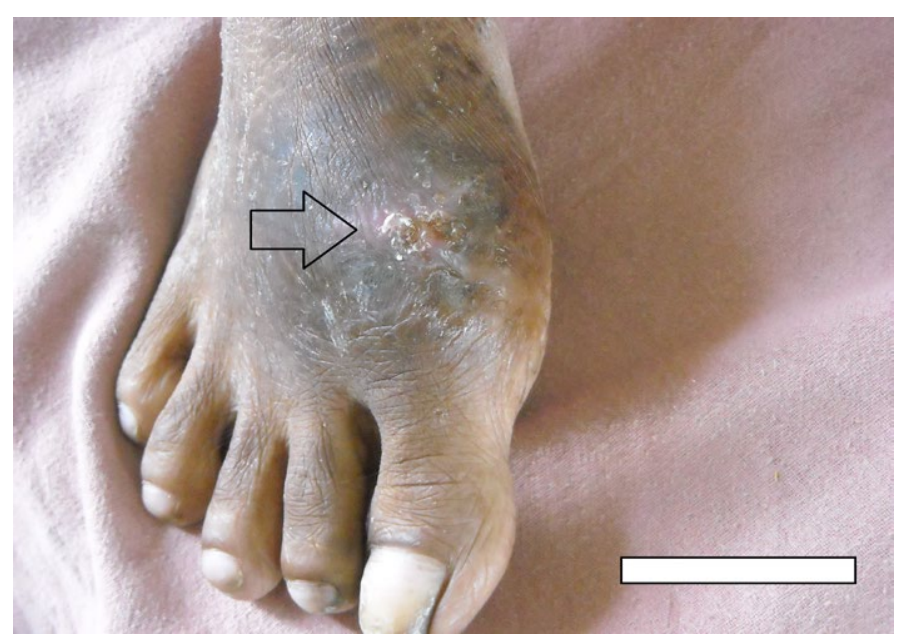

Figure 2. Lesion around the ball of great toe of right foot at presentation.

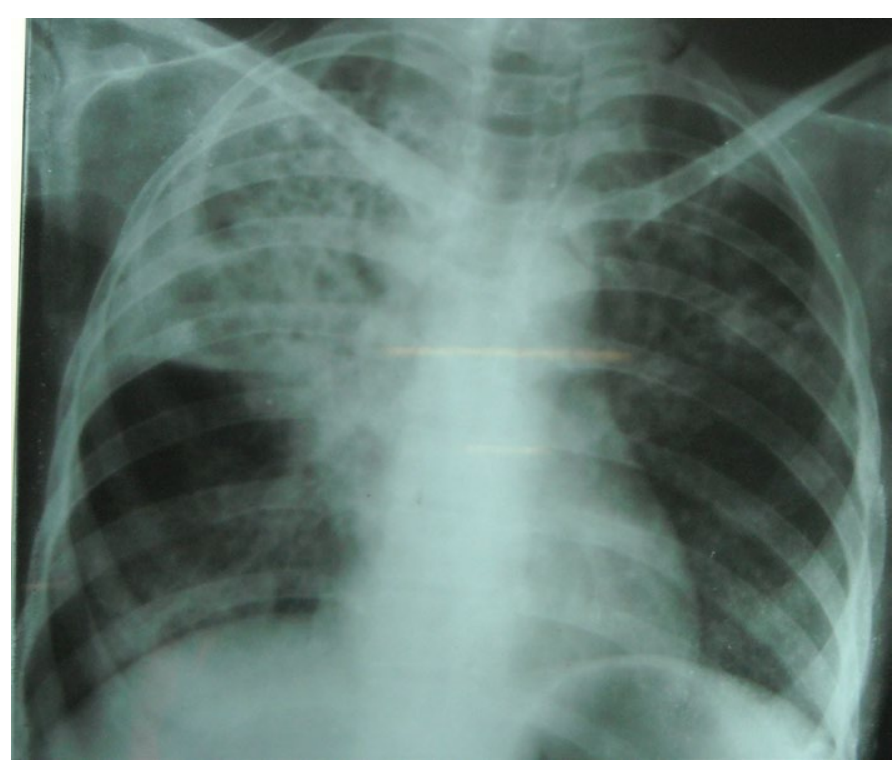

Figure 3. Chest X-ray at presentation, showing non-homogeneous opacity in right upper, middlezones and left upper zone.

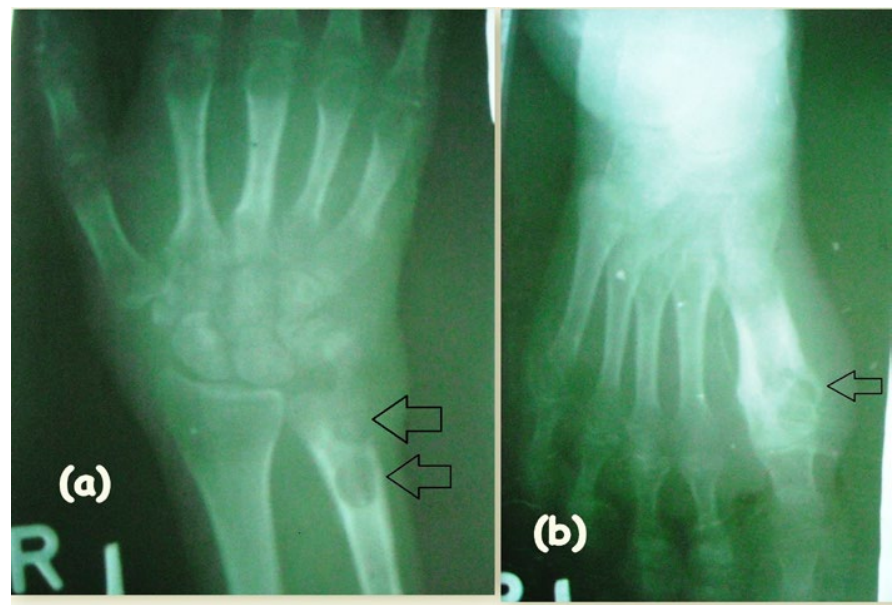

Figure 4. X-ray of (a) right hand and (b) right foot at presentation showing osteolytic lesions. swelling. Lesions of the hands (Figure 6a), foot (Figure 6b) and chest $\mathrm{x}$-ray (Figure 7) showed improvement. She was advised to continue the treatment for 12 months when co-trimoxazole and co-amoxiclav were stopped and sputum was found to be negative for both the organisms. Other medicines were continued for total duration of 24 months (to achieve a goal of 12 months of sputum negativity for $M$. abcessus). She is being followed up and is asymptomatic till date.

\section{Discussion}

Both Nocardia and NTM can cause chronic lung disease with fever, productive cough, weight loss and sometimes haemoptysis. Bones and joints are $2^{\text {nd }}$ most common extra-pulmonary sites to be infected with Nocardia. [5]. In addition to lung disease, chronic osteomyelitis of multiple small bones of hands and feet along with arthritis of elbow, wrist, ankle is uncommon in a single patient. Multiple osteomyelitic bony lesions due to Nocardia infection is also very rare in literature [5]. NTM usually involve single joint or bone after local trauma or surgical inoculation by infected instruments. Cluster of such cases can occur from health care settings where surgery or endoscopic procedures have taken place [6]. M. abscessus is the third most frequently recovered NTM causing respiratory illness in the United States. Most of the patients with this lung disease are white, female nonsmokers, and older than 60 years of age, with no predisposing conditions. Underlying disorders that are associated with the disease include bronchiectasis and prior mycobacterial infection. As with MAC lung disease, the origin of bronchiectasis in these patients may be the M. abscessus infection, but this is not known with certainty. Other rare predisposing conditions include gastroesophageal disorders with chronic vomiting,

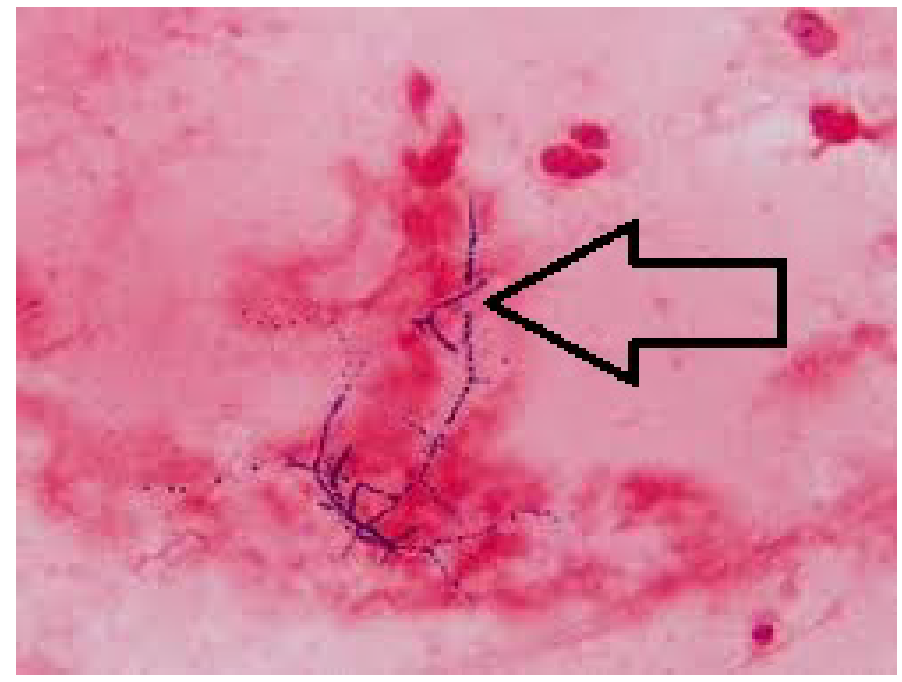

Figure 5. Gram stained smear of sputum showed gram positive beaded, branched organisms suggestive of nocardia.

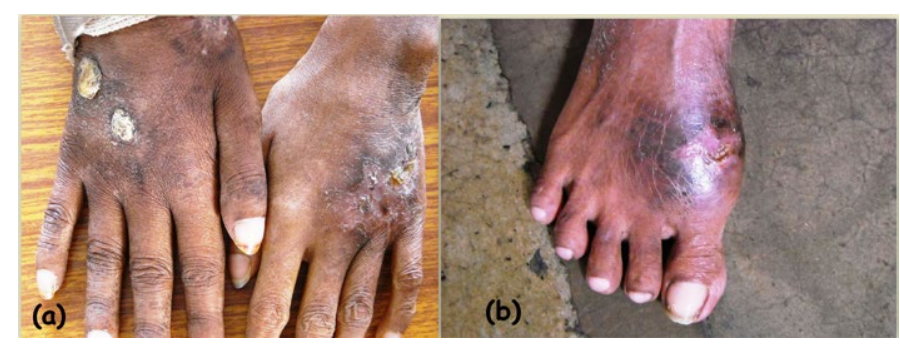

Figure 6. Lesions of (a) hands and (b) right foot after 1 month of treatment. 


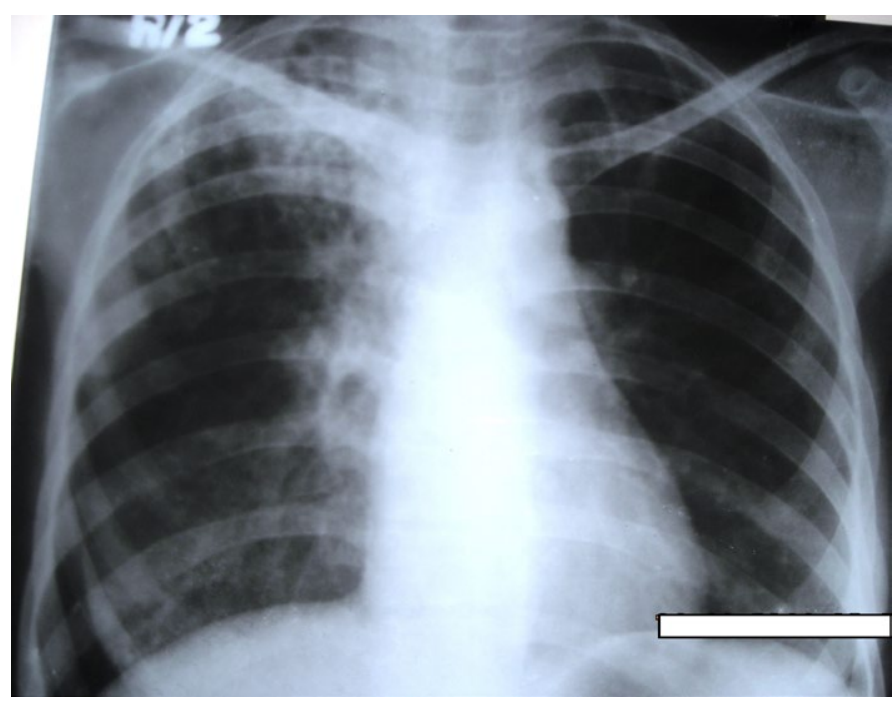

Figure 7. Chest X-ray after 1 month of treatment

lipoid pneumonia and cystic fibrosis [5]. No literature was found about Nocardia and NTM co-infection in a single patient. CT scan of brain did not reveal any pathology in our case, though, brain is the commonest extra-pulmonary site involved in nocardiosis [3].

\section{Conclusion}

The unique features of our case are as follows:

1) Co-infection by nocardia and non-tubercular mycobacteria in lungs,bones and joints is rare and not found in the literature

2) Simultaneous isolation of M.abscessus in sputum and osteomyelitic discharge is very uncommon.

\section{Conflict of interest}

None declared.

\section{References}

1. Lerner PI (1996) Nocardiosis. Clinical Infectious Diseases 22: 894-895

2. Falkinham JO (2002) Nontuberculous mycobacteria in the environment. Clin Chest Med 23:520-551.

3. Hoffman PC, Fraser DW, Rohicsek F, O’Bar PR, Mauney CU (1981) Two outbreaks of sternal wound infections due to organisms of the Mycobacterium fortuitum complex. $J$ Infect Dis 143:533-542. [Crossref]

4. Griffith DE, Aksamit T, Brown-Elliott BA, Catanzaro A, Daley C, et al. (2007) An Official ATS/IDSA Statement: Diagnosis, Treatment, and Prevention of Nontuberculous Mycobacterial Diseases. Am J RespirCrit Care Med 175: 367-416. [Crossref]

5. Harrison's Principles of Internal Medicine; Nocardiosis; Gregory A. Filice; (18thedn), 1323.

6. http://www.isradiology.org/tropical_deseases/tmcr/chapter6/clinical5.htm

Copyright: $\odot 2015$ Pal D. This is an open-access article distributed under the terms of the Creative Commons Attribution License, which permits unrestricted use, distribution, and reproduction in any medium, provided the original author and source are credited. 